\title{
Systematic Review of HIV Transmission between Heterosexual Serodiscordant Couples where the HIV- Positive Partner Is Fully Suppressed on Antiretroviral Therapy
}

\author{
Mona R. Loutfy ${ }^{1,2,3,4 *}$, Wei $\mathrm{Wu}^{1}$, Michelle Letchumanan ${ }^{1,3}$, Lise Bondy ${ }^{2}$, Tony Antoniou ${ }^{3,4}$, \\ Shari Margolese ${ }^{1}$, Yimeng Zhang ${ }^{2}$, Sergio Rueda ${ }^{5,10}$, Frank McGee ${ }^{6}$, Ryan Peck ${ }^{7}$, Louise Binder ${ }^{8}$, \\ Patricia Allard ${ }^{9}$, Sean B. Rourke ${ }^{4,5,10}$, Paula A. Rochon ${ }^{1,2,3}$
}

1 Women's College Research Institute, Women's College Hospital, University of Toronto, Toronto, Canada, 2 Faculty of Medicine, University of Toronto, Toronto, Canada, $\mathbf{3}$ Institute of Health Policy, Management and Evaluation, University of Toronto, Toronto, Canada, $\mathbf{4}$ St. Michael's Hospital, University of Toronto, Toronto, Canada, 5 Ontario HIV Treatment Network, Toronto, Ontario, Canada, 6 AIDS Bureau, Ontario Ministry of Health and Long Term Care, Toronto, Canada, 7 HIV and AIDS Legal Clinic Ontario, Toronto, Ontario, Canada, 8 Canadian Treatment Action Council, Toronto, Canada, 9 Canadian HIV/AIDS Legal Network, Toronto, Ontario, Canada, 10 Department of Psychiatry, University of Toronto, Toronto, Ontario, Canada

\begin{abstract}
Background: The risk of sexual HIV transmission in serodiscordant couples when the HIV-positive partner has full virologic suppression on combination antiretroviral therapy (CART) is debated. This study aims to systematically review observational studies and randomized controlled trials (RCTs), evaluating rates of sexual HIV transmission between heterosexual serodiscordant couples when the HIV-positive partner has full suppression on CART.

Methods and Findings: We searched major bibliographic databases to November 2012 for relevant observational studies and RCTs without language restrictions. Conference proceedings, key journals and bibliographies were also searched. Studies reporting HIV transmission rates, CART histories and viral loads of the HIV-positive partners were included. Two reviewers extracted methodologic characteristics and outcomes. Of 20,252 citations, 3 studies met all eligibility criteria with confirmed full virologic suppression in the HIV-positive partner. We included 3 additional studies (2 cohort studies, 1 RCT) that did not confirm viral suppression in the HIV-positive partner at transmission in a secondary meta-analysis. Methodologic quality was reasonable. The rate of transmission in the 3 studies confirming virologic suppression was 0 per 100 person-years $(95 \% \mathrm{Cl}=0-0.05)$, with low heterogeneity $\left(\mathrm{I}^{2}=0 \%\right)$. When we included the 3 studies that did not confirm virologic suppression, the rate of transmission was 0.14 per 100 person-years $(95 \% \mathrm{Cl}=0.04-0.31)\left(I^{2}=0 \%\right)$. In a sensitivity analysis including all 6 studies, the rate of transmission was 0 per 100 person-years $(95 \% \mathrm{Cl}=0-0.01)$ after omitting all transmissions with known detectable or unconfirmed viral loads, as full suppression in these cases was unlikely. Limitations included lack of data on same-sex couples, type of sexual intercourse (vaginal vs. anal), direction of HIV transmission, exact viral load at the time of transmission, sexually transmitted infections (STI) rates, and extent of condom use.

Conclusions: Our findings suggest minimal risk of sexual HIV transmission for heterosexual serodiscordant couples when the HIV-positive partner has full viral suppression on CART with caveats regarding information on sexual intercourse type, STIs, and condom use. These findings have implications when counseling heterosexual serodiscordant couples on sexual and reproductive health. More research is needed to explore HIV transmission risk between same-sex couples.
\end{abstract}

Citation: Loutfy MR, Wu W, Letchumanan M, Bondy L, Antoniou T, et al. (2013) Systematic Review of HIV Transmission between Heterosexual Serodiscordant Couples where the HIV-Positive Partner Is Fully Suppressed on Antiretroviral Therapy. PLoS ONE 8(2): e55747. doi:10.1371/journal.pone.0055747

Editor: Sarah Pett, University of New South Wales, Australia

Received June 19, 2012; Accepted December 31, 2012; Published February 13, 2013

Copyright: (c) 2013 Loutfy et al. This is an open-access article distributed under the terms of the Creative Commons Attribution License, which permits unrestricted use, distribution, and reproduction in any medium, provided the original author and source are credited.

Funding: This work was supported by grant \# KRS-102081 (www.cihr-irsc.gc.ca.). The funders had no role in study design, data collection and analysis, decision to publish, or preparation of the manuscript.

Competing Interests: Dr. MRL is a PLOS ONE Editorial Board member and this does not alter the authors' adherence to all the PLOS ONE policies on sharing data and materials.

*E-mail: mona.loutfy@wchospital.ca

\section{Introduction}

The risk of horizontal HIV transmission between serodiscordant couples when the HIV-positive partner has full viral suppression with combination antiretroviral therapy (cART) remains unclear [1-3]. This issue is of particular importance to longstanding monogamous discordant couples and to discordant couples considering conception through vaginal heterosexual intercourse [4-6]. In the midst of uncertainty regarding the risk of sexual HIV transmission in these contexts, the Swiss National AIDS Commission issued a statement in January 2008 specifying that HIVinfected individuals could be considered non-infectious if they met three conditions: adherence to cART under the care of an HIV 
physician, virologic suppression below the level of detection for at least six months, and absence of concomitant sexually transmitted infections (STIs) [7]. The "Swiss statement" was highly contentious, particularly in light of evidence demonstrating viral particles in the genital secretions of $5-48 \%$ of patients with undetectable plasma viremia [8-10].

Since the release of the Swiss Statement [7], 4 systematic reviews have been published quantifying the risk of sexual HIV transmission in serodiscordant couples [11-14]. Powers et al (2008) [11] and Boily et al (2009) [12] synthesized evidence on the rates of HIV transmission between HIV-positive individuals and uninfected partners per coital act, with a particular focus on contributing co-factors such as genital ulcer disease, circumcision, and stage of illness. However, neither review accounted for the impacts of antiretroviral use or the viral load of index cases on the risk of transmission. These limitations were somewhat addressed by Attia et al, who conducted a systematic review examining the role of cART use and viral load on HIV transmission between heterosexual discordant couples [13]. However, of the 5 studies included in their review, 3 were conference abstracts and the 2 studies with viral load data were not recent (from 2005 and 2008). The most recent systematic review, available in the Cochrane library examining the impact of cART on sexual transmission of HIV in serodiscordant couples, did not consider the influence of undetectable HIV plasma viral load on the risk of HIV transmission between discordant partners [14]. In addition, these reviews were published prior to the availability of data from the multicenter, randomized controlled trial (RCT), HIV Prevention Trials Network (HPTN) Study 052 [15]. In this trial of 1,763 serodiscordant couples, early initiation of cART was associated with a 96\% reduction in the number of linked HIV-1 transmissions relative to delayed cART (i.e. waiting to initiate cART when a clinical event occurred or CD4+ cell count reduced $<250$ cells/ $\mu \mathrm{L})$ [15].

In summary, existing systematic reviews neither address all factors that could modulate the risk of horizontal HIV transmission between heterosexual serodiscordant couples when the HIVpositive partner has full viral suppression on cART including HIV RNA levels, genital ulcer disease, male circumcision status, and hormonal contraceptive use, nor do they include the most up-todate evidence surrounding this topic $[11,15]$. Consequently, we undertook a systematic review and meta-analysis of quantitative observational studies and RCTs to quantify the risk of horizontal transmission between serodiscordant couples when the HIVpositive partner has full viral suppression on cART. We hypothesize that the risk of horizontal transmission between serodiscordant heterosexual couples when the HIV-positive partner has full viral suppression on cART will be extremely low.

\section{Methods}

\section{Protocol and Registration}

This project was not prospectively registered. A protocol was developed during the planning process (Grant\# KRS 102081).

\section{Literature Search Strategy}

This systematic review adheres to guidelines outlined in The Cochrane Group Handbook for Systematic Reviews of Interventions [16]. As well, the PRISMA flow diagram and checklist and MOOSE checklist were used [17,18]. In consultation with a librarian, a strategy (available in online appendix) was developed for searching MEDLINE (1950- November 2012), EMBASE (1980- November 2012), CINAHL (1980- November 2012), and Web of Science (2004- November 2012) for all relevant observational studies and RCTs regardless of country or language. Keywords searched included "Human immunodeficiency virus", "HIV transmission", "intercourse", and "antiretroviral therapy" (see Appendix for more detail). We also undertook a hand search of journals (online appendix) from June 2010 to November 2012 to identify articles missed by our search, and searched the electronic proceedings of the Conference on Retroviruses and Opportunistic Infections (2008 to 2011), International AIDS Conference (2008 and 2010), and International AIDS Society Conference (2009 and 2011 ) for relevant abstracts. As only published peer-reviewed full manuscripts were included, authors were contacted by email to ascertain if a full manuscript or publication was available. If any additional information or clarification was required, authors were also contacted.

\section{Study Selection}

Three reviewers divided and screened all citations from the literature search for obvious exclusions. The remaining abstracts were assessed independently by two reviewers (LB, ML), and disagreements were resolved by a third reviewer (MRL). We included published studies of serodiscordant heterosexual or samesex couples that provided data regarding all of the following: (1) sexual contact, (2) HIV-positive partner taking cART, (3) confirmed undetectable viral load at the time of the HIV transmission, and (4) reported HIV infections rates in HIVnegative partner. The definition of "undetectable viral load" consisted of the viral load being below the level of detection for the year that the test was carried out. This combination of data facilitated our aim to determine the rates of horizontal HIV transmission between serodiscordant couples when the HIVpositive partner has full viral suppression with cART. Studies that did not confirm index case viral loads at the time of transmission were considered for a secondary meta-analysis. As a final step, three experts in the area reviewed our final study selection to identify any missing literature (EM, RP, JM).

\section{Data Extraction}

Two reviewers (MRL, LB) designed and independently piloted a data extraction form. The following items were extracted: study information (location of study, methodology/type of study, study setting, enrolment period, total couples enrolled, total analyzed, follow-up duration, total follow-up in person-years), participants' demographic information (age, sexual orientation of HIV-positive individual, race/ethnicity), clinical HIV information (type of cART, frequency of HIV testing, frequency of viral load measurement, viral load limit, viral load of index partner, type of assay used), risk factors for HIV transmission (testing of STIs, male circumcision of HIV-positive partner and/or HIV-negative partner, \% condom use), and outcome measures reported (HIV transmission on and not on cART, overall HIV transmission rate per 100 person-years and per sexual act, and whether the transmitted virus was genotypically linked). Two investigators (LB, WW) independently assessed each full-text article and extracted the required data. The datasets were compared for each study and a third party settled disagreements (MRL).

\section{Risk of Bias Assessment}

The risk of bias of each eligible study was assessed by two investigators (LB, WW) using the 8-item Newcastle-Ottawa Scale for observational studies [19] (online Appendix) and the Cochrane Risk of Bias tool [20] for RCTs. The datasets were compared and a third party settled disagreements (MRL). These instruments are described in detail elsewhere $[19,20]$. 


\section{Outcome Measures and Data Synthesis}

The primary outcome of interest was incidence of HIV infection in the HIV-negative individual partnered with an HIV-positive person taking cART with fully suppressed viral load. When possible, it was planned to report on incidence of HIV infection per sexual act.

We reported on whether the index case was on cART, whether the HIV transmission occurred on cART, the viral load at the time of transmission, overall HIV transmission rate per 100 personyears, HIV transmission rate per 100 person-years for those on cART and those not on cART, all with $95 \%$ confidence intervals (CI[s]). If the exact follow-up time was not available, authors were contacted to attain this information; if there was no reply, it was estimated from the available data. If available, incidence of HIV infection per sexual act was also collected.

\section{Data Analysis}

An a priori decision was made to meta-analyze unadjusted data as adjustment of confounders in observational studies is rare or is inconsistently reported across studies [21]. A meta-analysis was performed of the data reported on the HIV transmission rate per 100 person-years using a fixed-effects Poisson regression model to report the summary HIV transmission rate and 95\% CI. Poisson meta-regression was analyzed using a Bayesian framework. Bayesian statistics uses probabilities to make inferences and makes use of new evidence to update prior information [22]. A second meta-analysis was conducted including studies where the HIVpositive partner was on cART with unconfirmed viral suppression at the time of transmission. For this second meta-analysis, the pooled odds ratio (OR) of HIV transmission (cART vs. no cART) was calculated and reported with the 95\% CI for eligible studies. Clinical heterogeneity of the studies was assessed and reported in the table of included studies. Statistical heterogeneity was assessed by calculating $\mathrm{I}^{2}$ values [23]. A sensitivity analysis using a randomeffects model was performed to determine the overall HIV transmission rate per 100 person-years with the $95 \%$ CI from only HIV-positive partners on cART with confirmed undetectable viral load. This analysis was carried out by including all studies (those with confirmed and known or unconfirmed suppressed viral loads) but omitting all transmissions with known unsuppressed or unconfirmed viral loads. The meta-analytic software, Comprehensive Meta-analysis Version 2 (Biostat, Englewood NJ [2005]) [24] and Winbugs software release Version 1.4 (Cambridge, UK [2003]) were used [25]. Markov Chains were initiated with three different sets of values and employed 20,000 iterations. The convergence of the model was assessed using the Gelman-RubinBrooke statistic $[24,25]$.

\section{Results}

After removal of duplicate references, 20,252 records from our search of electronic databases were identified, of which 145 were ultimately deemed potentially relevant and retrieved as full-length articles for detailed review (Figure 1). After excluding studies based on design and outcomes of interest, 5 studies from the literature search meeting all inclusion criteria were identified [26-30]. Three of the articles reported on the same cohort [28-30], leaving 3 studies for analysis. An additional 6 articles met criteria for our secondary meta-analysis and sensitivity analysis as they either had known unsuppressed viral loads or unconfirmed viral load suppression at the time of the transmission [15,31-35]. Three sets of 2 articles reported on the same cohorts, leaving an additional 3 studies for the secondary and sensitivity analyses. The details of the baseline characteristics of the included studies are reported in Table 1. No studies meeting our inclusion criteria provided enough data on same-sex couples, thereby limiting our findings to heterosexual serodiscordant couples. Specifically, there were only $3 \%$ same-sex couples in the HPTN 052 study (approximately 53) (gender unspecified). Furthermore, because insufficient data were provided regarding HIV transmission rates per coital act, all subsequent findings are reported by 100 personyears. Also, not enough data were available to present results on transmission rates through intercourse exclusively without condoms, by type of sexual act (vaginal vs. anal), correcting for presence of STIs, or by female-to-male or male-to-female transmission.

The 3 cohort studies with confirmed suppressed virus at the time of HIV transmission reported on 991 heterosexual couples from Brazil, Spain and Uganda with 2,064 person-years of follow up being available [25-29]. The additional 2 cohort studies with unconfirmed viral load at the time of HIV transmission were conducted in various countries in Africa and reported on 4,307 couples [31-34]. The HPTN 052 RCT was carried out in various African countries, India, Thailand, the United States of America, and Brazil and reported on 1,763 couples. There was 8170 personyears of follow-up for the latter 3 studies [15,31-35].

\section{Assessment of Risk of Bias and Data from Individual Studies}

The results of the risk of bias assessments are reported in Table 2 for the included observational studies and Table 3 for the RCT. Overall, all studies had low risk of bias.

The resulting data from each included study are presented in Table 4 . In the 3 cohort studies meeting all inclusion criteria, no transmissions were observed if the HIV-positive partner was taking cART and had undetectable viremia [26-30]. In contrast, the 3 studies that had known detectable or unconfirmed viral load data at the time of transmission to the HIVnegative partner recorded 4 such events [15,31-35]. One transmission occurred in each cohort study and 2 in the HPTN 052 study and all were genetically linked. [15,31-35]. Although insufficient detail regarding exact viral load at the time of the transmissions is available, it appears that these events occurred shortly following the initiation of treatment in all cases before full virologic suppression could be attained [15,31-36]. For example, Donnell and colleagues report a female-to-male transmission that occurred within 3 months of the HIV-infected partner initiating cART [31]. Similarly, the genetically linked transmissions from the HPTN 052 study [15,35] were identified 3 months and 4 weeks after the HIV-positive partner initiated cART, before there was complete viral suppression [15,30-35]. In some of the cases, the viral load was known to be detectable $[15,35,36]$.

\section{Meta-analysis and Heterogeneity Assessment}

The first meta-analysis included the 3 cohort studies with confirmed suppressed virus at the time of HIV transmission and is presented in Figure 2 [26-30]. The pooled summary HIV transmission rate from these 3 studies was 0 per 100 personyears $(95 \% \mathrm{CI}=0-0.05)$. The second meta-analysis included all 6 studies, regardless of whether virologic suppression was confirmed at the time of transmission (Figure 2) [15,26-35]. The pooled summary HIV transmission rate from these 6 studies was 0.14 per 100 person-years $(95 \% \mathrm{CI}=0.04-0.31)$. Five of these 6 studies reported on HIV transmission rates in those on and not on cART (all except Apondi et al), allowing a pooled OR to be calculated. For the HPTN 052 study, the authors were contacted and provided the person-year follow up 


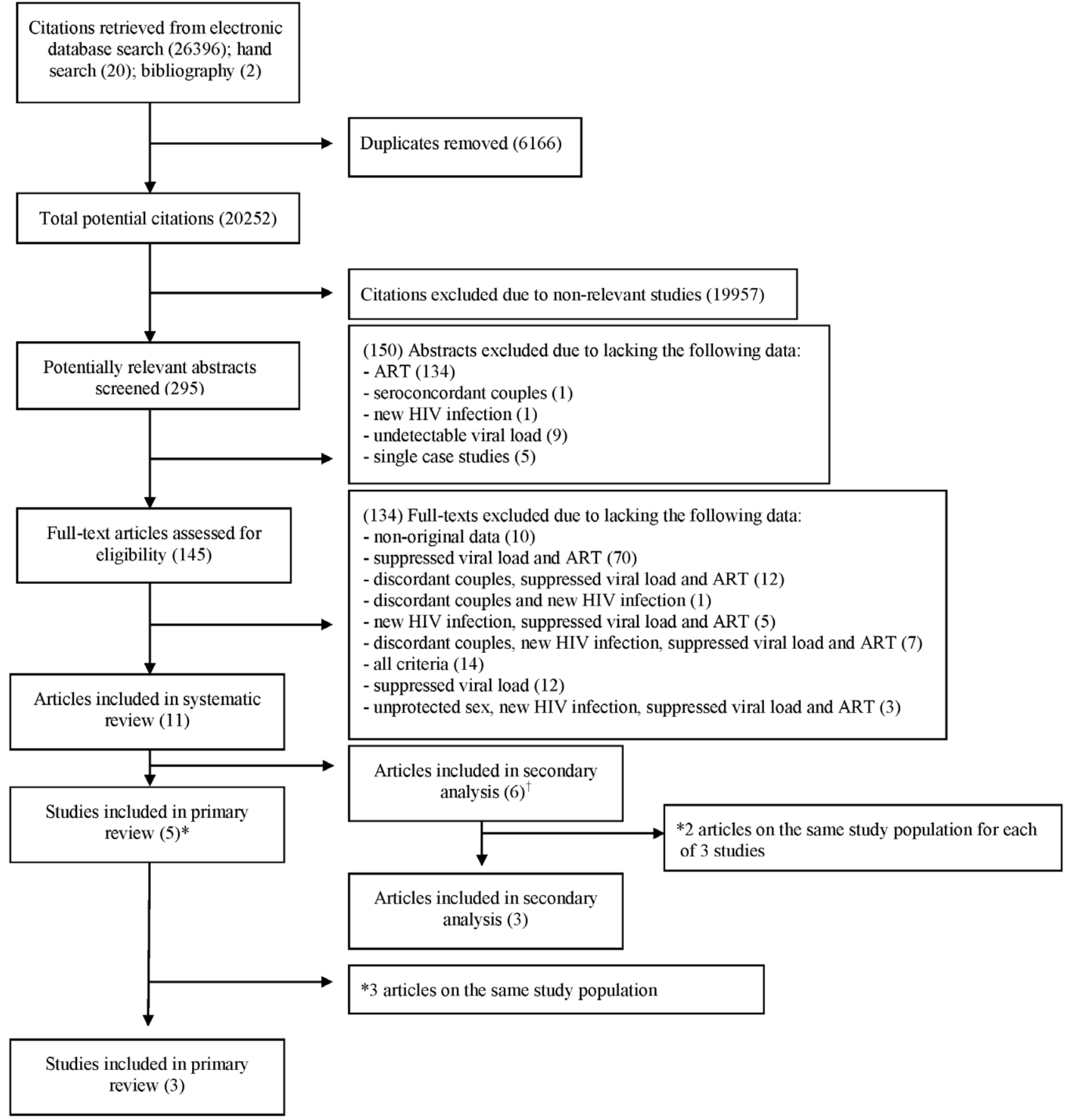

Figure 1. Flow diagram (selection strategy) of included studies. doi:10.1371/journal.pone.0055747.g001

values for the participants in the delayed treatment group who did and did not receive cART [37]. The pooled OR for HIV transmission per 100 person-years for those on versus not on cART from the 5 studies was $0.05(95 \% \mathrm{CI}=0.01-0.17)$.

Clinical heterogeneity among the studies is described in Table 1. Statistical heterogeneity was identified for the two meta-analyses and was $0 \%$ for both.

\section{Sensitivity Analysis}

As the data suggest that the 4 transmissions for the studies with unconfirmed viral suppression at the time of HIV transmission occurred when the viral load was either known or likely to be unsuppressed [15,31-36], a sensitivity analysis was carried out for the pooled HIV transmission rate per 100 person-years from all HIV-positive partners on cART from the 6 studies excluding these 4 transmissions and is presented in Figure 2. The pooled summary HIV transmission rate from this sensitivity analysis was 0 per 100 person-years $(95 \% \mathrm{CI}=0-0.01)$. 


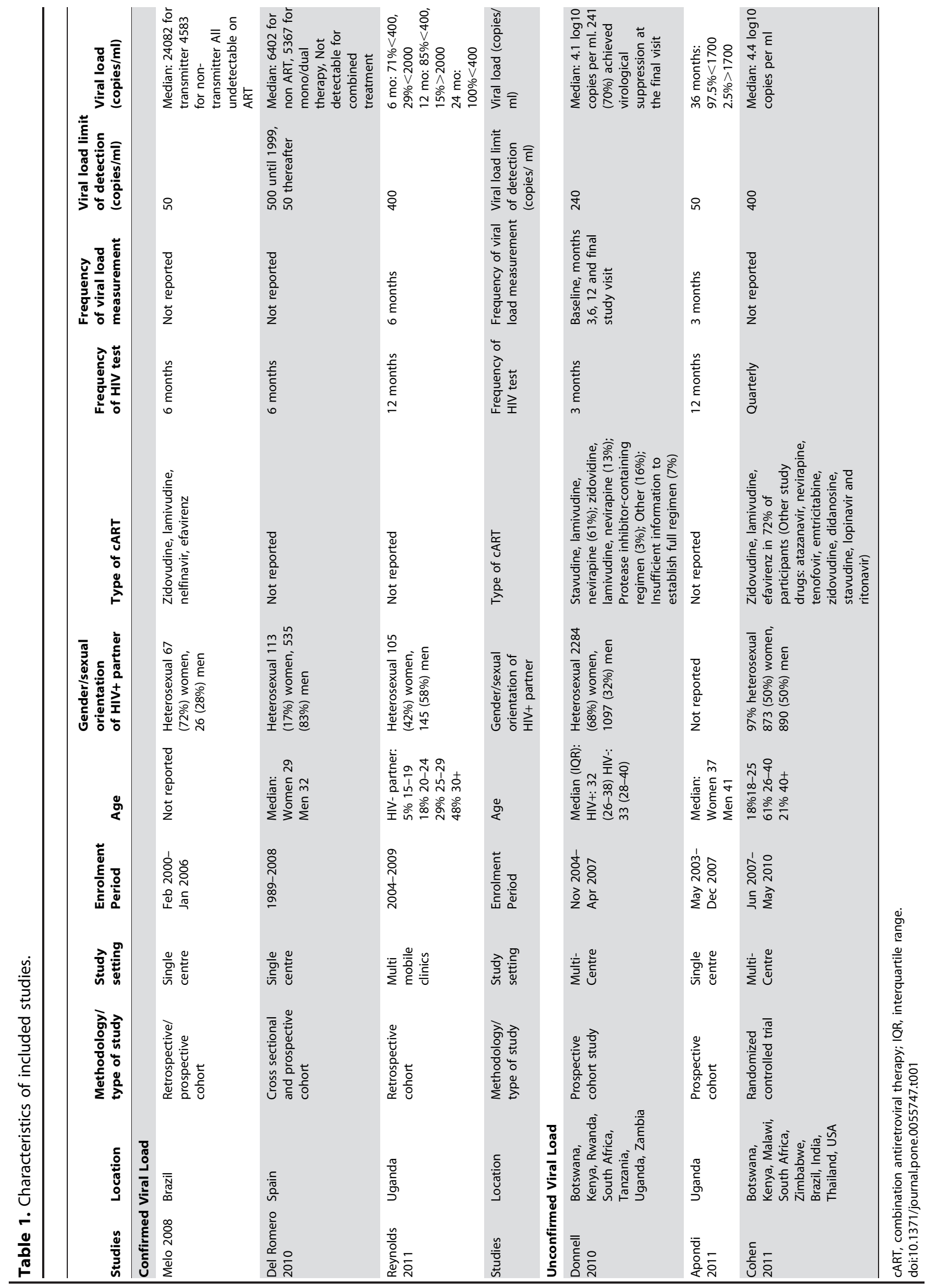




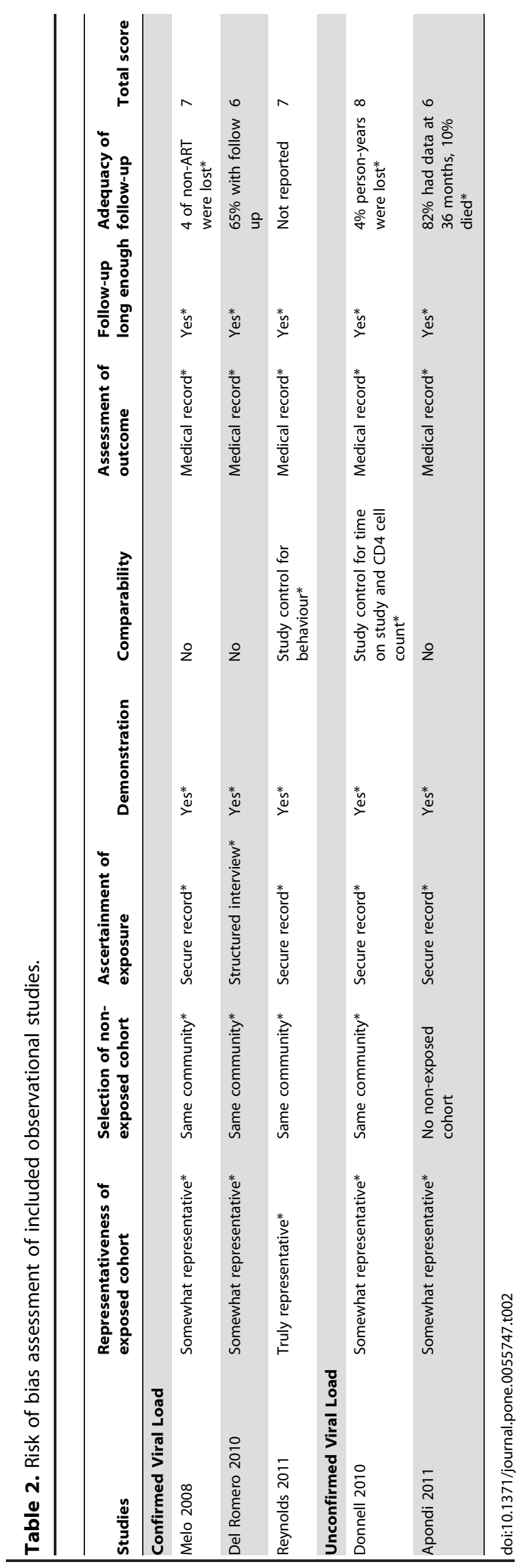

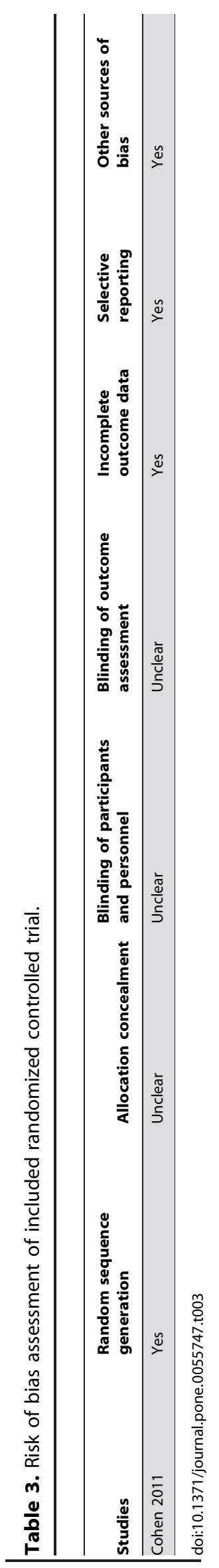

February 2013 | Volume 8 | Issue 2 | e55747 


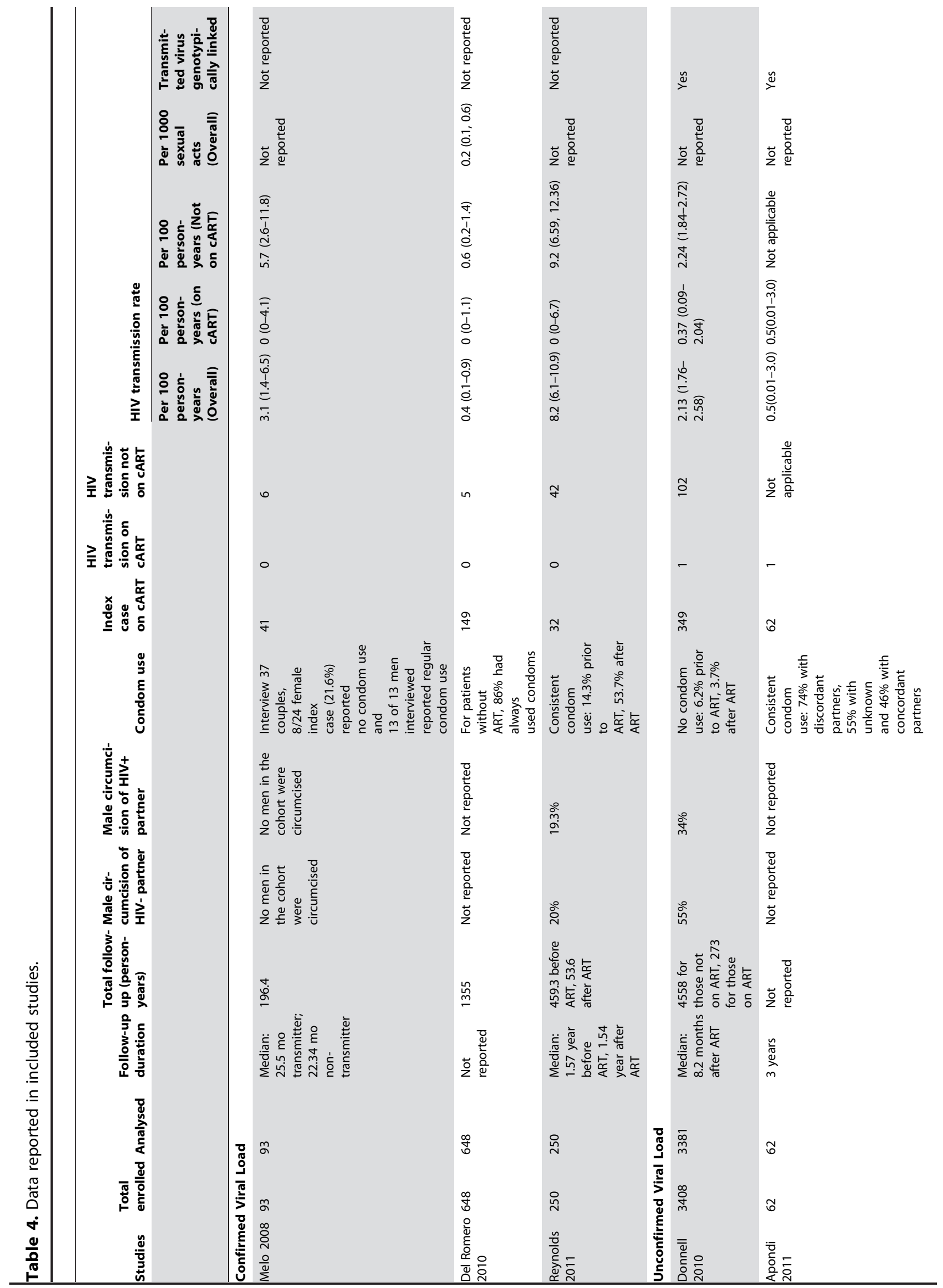




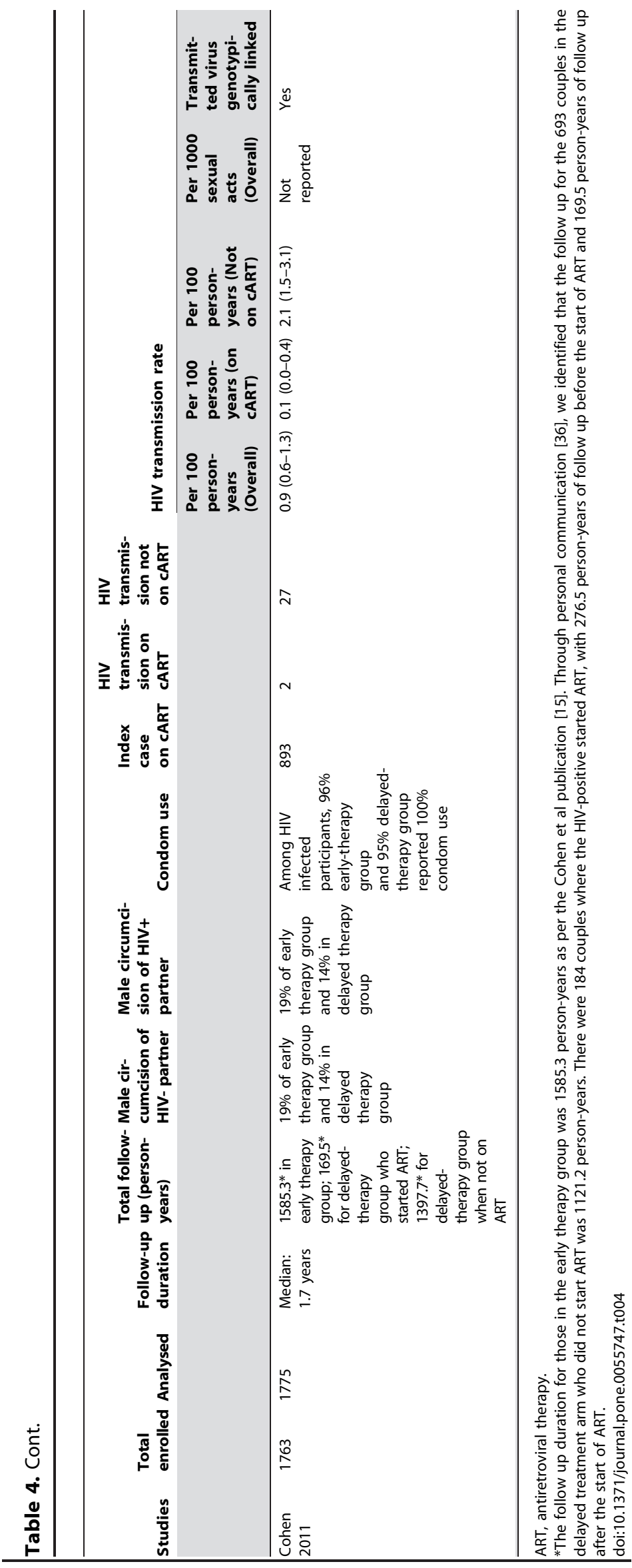


Forest plot of HIV transmisssion rates per 100 person-years of confirmed and unconfirmed viral loads

Study name

$\begin{array}{lcccc} & \begin{array}{l}\text { Event } / \\ \text { person years }\end{array} & \text { Rate } & \begin{array}{c}\text { Lower } \\ \text { limit }\end{array} & \begin{array}{c}\text { Upper } \\ \text { limit }\end{array} \\ \begin{array}{l}\text { Confirmed viral load } \\ \text { Melo 2008 }\end{array} & 0 / 90 & 0.00 & 0.00 & 4.02 \\ \text { Del Romero 2010 } & 0 / 492 & 0.00 & 0.00 & 0.75 \\ \text { Reynolds 2011 } & 0 / 53 & 0.00 & 0.00 & 6.72 \\ \text { Subtotal } & & 0.00 & 0.00 & 0.05 \\ \text { Unconfirmed viral load } & & & & \\ \text { Donnell 2010 } & 1 / 273 & 0.36 & 0.01 & 2.02 \\ \text { Apondi 2011 } & 1 / 185 & 0.54 & 0.01 & 2.97 \\ \text { Cohen 2011 } & 2 / 1755 & 0.11 & 0.01 & 0.41 \\ \text { Subtotal } & & 0.18 & 0.05 & 0.40 \\ \text { Total } & & 0.14 & 0.04 & 0.31\end{array}$

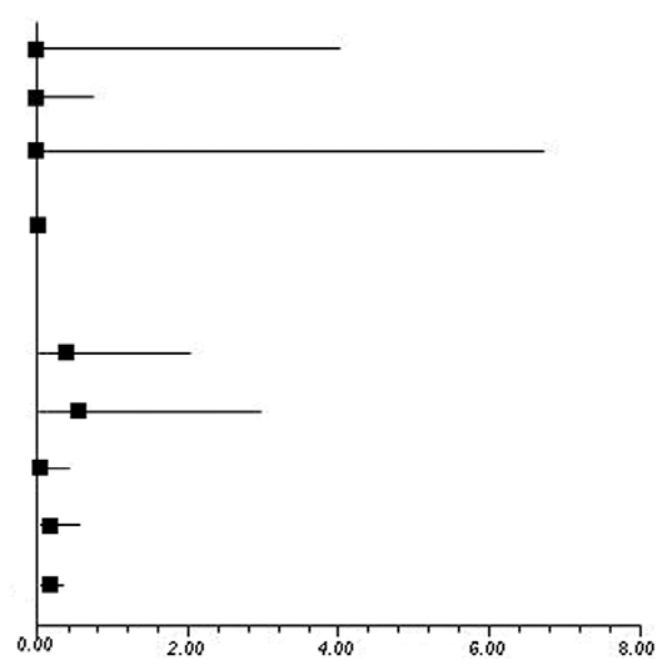

Forest plot of HIV transmission rates per 100 person-years, excluding unconfirmed viral loads

\begin{tabular}{lcccc} 
Study name & \multicolumn{3}{c}{ Statistics for each study } & \\
\cline { 3 - 4 } & $\begin{array}{l}\text { Event } / \\
\text { person years }\end{array}$ & Rate & $\begin{array}{c}\text { Lower } \\
\text { limit }\end{array}$ & $\begin{array}{c}\text { Upper } \\
\text { limit }\end{array}$ \\
Melo 2008 & $0 / 90$ & 0.00 & 0.00 & 4.02 \\
Del Romero 2010 & $0 / 492$ & 0.00 & 0.00 & 0.75 \\
Reynolds 2011 & $0 / 53$ & 0.00 & 0.00 & 6.72 \\
Donnell 2010 & $0 / 273$ & 0.00 & 0.00 & 1.34 \\
Apondi 2011 & $0 / 185$ & 0.00 & 0.00 & 1.97 \\
Cohen 2011 & $0 / 1755$ & 0.00 & 0.00 & 0.21 \\
Total & & 0.00 & 0.00 & 0.01
\end{tabular}

Figure 2. Forest plots of HIV transmission rates per 100 person-years with and without transmissions with unconfirmed viral suppression at the time of suppression. Footnote: The first forest plot is the summary of HIV transmission rates per 100 person-years with $95 \%$ confidence interval for 6 studies with confirmed and unconfirmed viral suppression at time of transmission. The second forest plot is the sensitivity analysis of the 6 studies with confirmed and unconfirmed viral suppression at the time of transmission with forest plot of the summary of HIV transmission rates per 100 person-years with $95 \%$ confidence interval reporting on HIV transmission when HIV-positive partner on combination antiretroviral therapy had confirmed viral suppression, omitting transmissions occurring with known or unconfirmed detectable viral loads at the time of transmission (i.e. 3 studies had 4 transmissions with known or unconfirmed detectable viral loads and these transmissions were excluded, while leaving the rest of data in the analysis).

doi:10.1371/journal.pone.0055747.g002

\section{Discussion}

We performed a comprehensive systematic review of the literature and meta-analyses to assess the risk of HIV sexual transmission between serodiscordant heterosexual partners when the HIV-positive partner has a confirmed undetectable plasma viral load with cART. In our review, only 3 studies where the HIV-positive partner had confirmed full viral suppression on cART were identified [26-30], with a pooled transmission rate of
0 per 100 person-years $(95 \% \mathrm{CI}=0-0.05)$. An additional 3 studies had important data to contribute with a total of 4 transmission events between serodiscordant partners, all occurring early after the start of cART and had known or likely declining viral load but were still detectable [15,31-35]. When included in the metaanalysis, the pooled HIV transmission rate was 0.14 per 100 person-years $(95 \% \mathrm{CI}=0.04-0.31)$. However, when these transmissions were omitted when viral suppression was unconfirmed in a sensitivity analysis, as detectable virus was known or likely, the 
pooled HIV transmission rate was 0 per 100 person-years $(95 \%$ $\mathrm{CI}=0-0.01)$. These findings are useful for the counseling of heterosexual couples considering unprotected intercourse as part of a monogamous consensual relationship [38,39]. Important caveats exist to our findings including the lack of data on same-sex couples, type of sexual intercourse (vaginal vs. anal), number of sexual encounters in a time period, direction of HIV transmission (male-to-female vs. female-to-male), exact viral load at the time of transmission, STI rates, and extent of condom use. In the counseling setting, it may be difficult to conceptualize the risk per 100 person-years as compared to a person's lifetime risk of HIV acquisition, which is much more tangible. An HIV transmission rate per 100 person-years can be converted to lifetime risk by multiplying the upper end of our 95\% CI (which was 0.0001 in our sensitivity analysis) by 20 to 50 years which is the expected life expectancy of someone diagnosed to be HIV positive who starts on cART $[39,40]$. This would translate into approximately a 1 in 204 to 1 in 50 lifetime risk of HIV transmission to the uninfected partner (i.e. $2-5 \%$; which is equivalent to $1 \%$ risk per 10 years of relationship and sexual activity).

Our review has several clinical implications. Specifically, our findings emphasize that early initiation of cART is a viable strategy for protection against HIV transmission at the individual level when the viral load is undetectable. Furthermore, our findings can further inform evidence-based discussions between fertility counselors and serodiscordant heterosexual couples $[38,39]$. While other groups have performed similar systematic reviews on the topic of HIV sexual transmission between serodiscordant heterosexual partners, our review and metaanalyses underline the importance of viral load in determining sexual HIV transmission in serodiscordant couples. HIV transmission in the studies we analyzed occurred when the participants were not taking cART, had detectable plasma HIV-1 viral load, or were in the window period between initiating cART and having a confirmed suppressed viral load. Powers et al (2008) [11] and Boily et al (2009) [12] did not report critical factors such as viral load and the influence of cART. Attia et al (2009) [13] integrated published and unpublished literature in their investigation. Based on two studies, they reported zero episodes of seroconversion from cART-treated sero-positive partners whose viral loads were $<400$ copies/ml and reported one transmission per 79 person-years as the upper limit of the 95\% CI with 291 person-years of follow-up [13]. In their review, a $92 \%$ reduction rate of HIV transmission was established when comparing cART-exposed index cases relative to those not on cART [13]. However, their rates included studies only presented in conference abstract form and from studies not updated past 2008 [13]. Anglemeyer et al (2011) reported 71 out of 436 episodes of transmission amongst couples treated with cART, but did not indicate index case viral loads to be able to comment on the role of suppressed viral load during these transmissions [14]. In a similar fashion to how Attia et al reported, the upper limit of the 95\% CI for our sensitivity analysis revealed one transmission in 1,000 person-years from data with 2,975 person-years of follow-up, more person-years being reported on the topic to date.

Our systematic review's findings do not contradict the Swiss National AIDS Commission statement that HIV-positive individuals are sexually non-infectious if they are fully virologically suppressed for at least 6 months with consistent adherence to cART and if no STIs are present [7]. Our review found that the rate of HIV-1 transmission for cART-treated patients with documented suppressed plasma viral load was 0 per 100-person years. However, our data only applies to heterosexual couples with some degree of variable condom use and does not incorporate impact of STIs. As published studies in same-sex couples were done using modeling and not with prospective data collection, our results must be used with caution and can only be inferred for this population.

Again, several limitations of our systematic review merit emphasis. Most notably, the impact of consistent condom use on the rate of HIV transmission could not be accounted for by most of the studies included in our review. In addition, most studies did not provide data regarding the presence of concomitant STIs, the prevalence of hormonal contraceptive use, and the risk of HIV transmission per coital act. Furthermore, extrapolation of these findings to same-sex couples is limited. The studies which have been conducted to date are of limited treatment length of time and do not account for durability of viral suppression. Also, type of sexual intercourse (vaginal vs. anal), number of sexual encounters in a time period, and direction of HIV transmission (male-tofemale vs. female-to-male) were not reported. These limitations must be considered when applying these findings to the clinical setting, and highlight additional areas where further research is required.

Again, a notable limitation of our review is the lack of data provided on details of exact condom use. This has made it impossible to calculate risk through intercourse exclusively without condoms. In the HPTN 052 study, $5 \%$ and $6 \%$ of the HIVpositive and HIV-negative participants, respectively, reported unprotected intercourse in the week prior to enrolment [15]. This variable of self-reported condom use $<100 \%$ at baseline was associated with significant increased transmission [15], however details are not provided.

Other important limitation of our study include that 1 ) the exact viral load of the HIV-positive partner at the time of transmission is difficult to capture as it is impractical to carry out daily viral load testing. Therefore, our selection criteria 'confirmed undetectable viral load at the time of the HIV transmission" poses some methodological difficulty as the number of studies with the known viral load of the HIV-positive partner at the time of transmission is low. This has led us to a bias of including studies where there was no HIV transmission in our primary analysis. Viral suppression and time of infection are time-varying variables that can only ever be measured at discrete time and rather should be thought of as observed viral suppression at the beginning and end of a time interval around the occurrence of a new infection; 2) the studies included in our systematic review did not include data on genital viral load. However, it can be deduced based on previous evidence that $5-48 \%$ of the HIV-positive patients with fully suppressed viral loads have detectable HIV-1 RNA in their genital secretion [810]. It has been the subject of controversy whether the presence of such genital HIV-1 RNA possesses any clinical significant risk to transmission. From our review, it appears to have had no influence on the results and did not lead to HIV transmission in the studies under review; and 3) there was a lack of data on STI rates and the impact of the presence of STIs. In summary, the limitations listed above culminate in the weakness that our study was unadjusted, which is understandably a difficult procedure in such analyses.

\section{Conclusions}

In summary, our systematic review contributes to the emerging body of literature expanding on the position put forth by the Swiss National AIDS Commission [7] that unprotected sexual intercourse is a viable conception and sexual option for heterosexual serodiscordant couples in monogamous relationships if the HIVinfected partner has full virologic suppression on cART, where both parties understand the limitations of the available data. Our research is important given the increasing importance of 
parenthood and healthy sexuality on the overall well-being of the lives of HIV-positive individuals and their partners [41-45]. Additional research is required to clarify the impact of cART on HIV transmission in serodiscordant same-sex couples and the impact of STIs, type of sexual intercourse and actual transmission rate with confirmed unprotected intercourse.

\section{Acknowledgments}

We thank the following for their assistance: Angela Eady, the librarian, for conducting our electronic database search; Elena Ivanova, for assistance with review of studies; Eric Mykhalovskiy, $\mathrm{PhD}$, for thoughtful critique of

\section{References}

1. Dieffenbach CW (2012) Preventing HIV transmission through antiretroviral treatment-mediated virologic suppression: aspects of an emerging scientific agenda. Curr Opin HIV AIDS 7: 106-110.

2. Cohen MS, McCauley M, Gamble TR (2012) HIV treatment as prevention and HPTN 052. Curr Opin HIV AIDS 7: 99-105.

3. El-Sadr WM, Coburn BJ, Blower S (2011) Modeling the impact on the HIV epidemic of treating discordant couples with antiretrovirals to prevent transmission. AIDS 25: 2295-2299.

4. Guthrie BL, Choi RY, Liu AY, Mackelprang RD, Rositch AF, et al. (2011) Barriers to antiretroviral initiation in HIV-1-discordant couples. J Acquir Immune Defic Syndr 58: e87-93.

5. Gray RH, Wawer MJ, Brookmeyer R, Sewankambo NK, Serwadda D, et al. (2001) Probability of HIV-1 transmission per coital act in monogamous, heterosexual, HIV-1-discordant couples in Rakai, Uganda. Lancet 357: 1149 1153.

6. Ruzagira E, Wandiembe S, Abaasa A, Bwanika AN, Bahemuka U, et al. (2011) HIV incidence and risk factors for acquisition in HIV discordant couples in Masaka, Uganda: an HIV vaccine preparedness study. PloS one 6: e24037.

7. Vernazza P, Hirschel B, Bernasconi E, Flepp M (2009) Les personnes séropositives ne souffrant d'aucune autre MST et suivant un traitment antirétroviral efficace ne transmettent pas le VIH par voie sexuelle. BMS 89: $165-169$.

8. Marcelin AG, Tubiana R, Lambert-Niclot S, Lefebvre G, Dominguez S, et al. (2008) Detection of HIV-1 RNA in seminal plasma samples from treated patients with undetectable HIV-1 RNA in blood plasma. AIDS 22: 1677-1679.

9. Sheth PM, Kovacs C, Kemal KS, Jones RB, Raboud JM, et al. (2009) Persistent HIV RNA shedding in semen despite effective antiretroviral therapy. AIDS 23: 2050-4.

10. Sheth PM, Yi TJ, Kovacs C, Kemal KS, Jones RB, et al. (2012) Mucosal correlates of isolated HIV semen shedding during effective antiretroviral therapy. Mucosal Immunol. doi: 10.1038/mi.2012.1. [Epub ahead of print].

11. Powers KA, Poole C, Pettifor AE, Cohen MS (2008) Rethinking the heterosexual infectivity of HIV-1: a systematic review and meta-analysis. Lancet 8: 553-563.

12. Boily MC, Baggaley RF, Wang L, Masse B, White RG, et al. (2009) Heterosexual risk of HIV-1 infection per sexual act: systematic review and meta-analysis of observational studies. Lancet 9: 118-129.

13. Attia S, Egger M, Muller M, Zwahlen M, Low N (2009) Sexual transmission of HIV according to viral load and antiretroviral therapy: systematic review and meta-analysis. AIDS 23: 1397-1404.

14. Anglemyer A, Rutherford GW, Baggaley RC, Egger M, Siegfried N (2011) Antiretroviral therapy for prevention of HIV transmission in HIV-discordant couples. Cochrane Database Syst Rev 8: CD009153.

15. Cohen MS, Chen YQ, McCauley M, Gamble T, Hosseinipour MC, et al. (2011) Prevention of HIV-1 infection with early antiretroviral therapy. NEJM 365: 493-505.

16. Higgins JPT, Altman DG, Sterne JAC (2011). Chapter 8: Assessing risk of bias in included studies. In: Higgins JPT, Green S, editors. Cochrane Handbook for Systematic Reviews of Interventions Version 5.1.0. Available: http://www. cochrane-handbook.org. Accessed 2012 Jan 4.

17. Liberati A, Altman DG, TetzlaffJ, Mulrow C, Gøtzsche PC, et al. (2009) The PRISMA statement for reporting systematic reviews and meta- analyses of studies that evaluate healthcare interven- tions: explanation and elaboration. BMJ 339: b2700.

18. Stroup DF, Berlin JA, Morton SC, Olkin I, Williamson GD, et al. (2000) Metaanalysis Of Observational Studies in Epidemiology (MOOSE) group. Metaanalysis of observational studies in epidemiology: a proposal for reporting. JAMA 283: 2008-2012.

19. Stang A (2010) Critical evaluation of the Newcastle-Ottawa scale for the assessment of the quality of nonrandomized studies in meta-analyses. Eur J Epidemiol 25: 603-605.

20. Higgins JP, Altman DG, Gotzsche PC, Jüni P, Moher D, et al. (2011) The Cochrane Collaboration's tool for assessing risk of bias in randomised trials. BMJ 343: d5928. the legal implications of our findings; James Wilton from the Canadian AIDS Treatment Information Exchange and Cecile Kazatchkine from the Canadian HIV/AIDS Legal Network for generous commentary on the manuscript.

\section{Author Contributions}

Contributed to manuscript editing: MRL ML YZ SM WW SR FM RP LB TA PA SR PR. Conceived and designed the experiments: MRL YZ SM SR FM RP LB TA PA SR PR. Performed the experiments: MRL LB ML YZ. Analyzed the data: WW MRL LB. Contributed reagents/materials/ analysis tools: WW MRL LB. Wrote the paper: MRL.

21. Peters J, Mengersen K (2008) Selective reporting of adjusted estimates in observational epidemiology studies: reasons and implications for meta-analyses. Eval Health Prof 31: 370-389.

22. Spiegelhalter DJ, Myles JP, Jones DR, Abrams KR (1999) Methods in health service research. An introduction to bayesian methods in health technology assessment. BMJ 319: 508-12.

23. Higgins JP, Thompson SG (2002) Quantifying heterogeneity in a meta-analysis. Stat Med 21: 1539-1558.

24. Borenstein M, Rothstein D, Cohen J (2000) Comprehensive Meta-analysis: A Computer Program for Research Synthesis. Englewood, NJ: Biostat; 25.

25. LunnDJ ThomasA, BestN SpiegelhalterD (2000) WinBUGS - a Bayesian modelling framework: concepts, structure, and extensibility. Statistics and Computing, 10: 325-337.

26. Melo MG, Santos BR, De Cassia Lira R, Varella IS, Turella ML, et al. (2008) Sexual transmission of HIV-1 among serodiscordant couples in Porto Alegre, southern Brazil. Sex Transm Dis 35: 912-915.

27. Reynolds SJ, Makumbi F, Nakigozi G, Kagaayi J, Gray RH, et al. (2011) HIV-1 transmission among HIV-1 discordant couples before and after the introduction of antiretroviral therapy. AIDS. 25: 473-477.

28. Del Romero J, Castilla J, Hernando V, Rodríguez C, García S (2010) Combined antiretroviral treatment and heterosexual transmission of HIV-1: cross sectional and prospective cohort study. BMJ 340: c2205.

29. Baggaley RF, Fraser G (2010) Modelling sexual transmission of HIV: testing the assumptions, validating the predictions. Curr Opin HIV AIDS 5: 269-276.

30. Wawer MJ, Gray RH, Sewankambo NK, Serwadda D, Li X, et al. (2005) Rates of HIV-1 transmission per coital act, by stage of HIV-1 infection, in Rakai, Uganda. J Infect Dis 191: 1403-1409.

31. Donnell D, Baeten JM, Kiarie J, Thomas KK, Stevens W, et al. (2010) Heterosexual HIV-1 transmission after initiation of antiretroviral therapy: a prospective cohort analysis. Lancet 375: 2092-2098.

32. Apondi R, Bunnell R, Ekwaru JP, Moore D, Bechange S, et al. (2011) Sexual behavior and HIV transmission risk of Ugandan adults taking antiretroviral therapy: 3 year follow-up. AIDS 25: 1317-1327.

33. Kravcik S, Victor G, Houston S, Sutherland D, Garber GE, et al. (1998) Effect of antiretroviral therapy and viral load on the perceived risk of HIV transmission and the need for safer sexual practices. J Acquir Immune Defic Syndr Hum Retrovirol 19: 124-129.

34. Suarez TP, Kelly JA, Pinkerton SD, Stevenson YL, Hayat M, et al. (2001) Influence of a partner's HIV serostatus, use of highly active antiretroviral therapy, and viral load on perceptions of sexual risk behavior in a community sample of men who have sex with men. J Acquir Immune Defic Syndr 28: 471477.

35. Eshleman SH, Hudelson SE, Redd AD, Wang L, Debes R, et al. (2011) Analysis of genetic linkage of HIV from couples enrolled in the HIV Prevention Trials Network 052 trial. J Infect Dis 204: 1918-1926.

36. Mastro TD, Cohen MS, Rees H (2011) Antiretrovirals for safer conception for HIV-negative women and their HIV-1-infected male partners: how safe and how available? AIDS 25(16): 2049-2051.

37. Personal communication with Drs Lei Wang and Myron S Cohen (permission obtained).

38. Panel on Treatment of HIV-Infected Pregnant Women and Prevention of Perinatal Transmission (2005) Recommendations for Use of Antiretroviral Drugs in Pregnant HIV-1-Infected Women for Maternal Health and Interventions to Reduce Perinatal HIV Transmission in the United States. AIDSinfo. Available: http://www.health.state.mn.us/divs/idepc/diseases/hiv/ perinatalguideline.pdf. Accessed 2012 Feb 24.

39. Ahmed A, Kaplan M, Symington A, Kismodi E (2011) Criminalizing consensual sexual behaviour in the context of HIV: consequences, evidence, and leadership. Glob Public Health 6 Suppl 3: S357-369.

40. Antiretroviral Therapy Cohort Collaboration (2008) Life expectancy of individuals on combination antiretroviral therapy in high-income countries: a collaborative analysis of 14 cohort studies. Lancet 372(9635): 293-9.

41. Hogg RS, Samji H, Cescon A, Modur SP, Napravnik S, et al. (2012) Temporal changes in life expectancy of HIV-positive persons in North America. 19th Conference on Retroviruses and Opportunistic Infections (CROI). Seattle WA, USA, March 5-8: abstract 137 (oral presentation). 
42. Loutfy MR, Hart TA, Mohammed SS, Su D, Ralph ED, et al. (2009) Fertility desires and intentions of HIV-positive women of reproductive age in Ontario, Canada: a cross-sectional study. PloS one 4: e7925.

43. Mantell JE, Smit JA, Stein ZA (2009) The right to choose parenthood among HIV-infected women and men. J Public Health Policy 30: 367-378.
44. Gupta GR (2000) Gender, sexuality, and HIV/AIDS: the what, the why, and the how. Can HIV AIDS Policy Law Rev 5: 86-93.

45. Remien RH, Carballo-Dieguez A, Wagner G (1995) Intimacy and sexual risk behaviour in serodiscordant male couples. AIDS Care 7: 429-438. 